\title{
COMMENT
}

Check for updates

\section{Endocrine and metabolic link to coronavirus infection}

\author{
Stefan R. Bornstein ${ }^{1,2,3 凶}$, Rinkoo Dalan ${ }^{4,5}$, David Hopkins ${ }^{6}$, Geltrude Mingrone $e^{2,7,8}$ \\ and Bernhard O. Boehm ${ }^{4,5}$
}

Type 2 diabetes mellitus and hypertension are the most common comorbidities in patients with coronavirus infections. Emerging evidence demonstrates an important direct metabolic and endocrine mechanistic link to the viral disease process. Clinicians need to ensure early and thorough metabolic control for all patients affected by COVID-19.

Type 2 diabetes mellitus (T2DM) seems to be a risk factor for acquiring the new coronavirus infection. Indeed, T2DM and hypertension have been identified as the most common comorbidities for other coronavirus infections, such as severe acute respiratory syndrome (SARS) and Middle East respiratory syndrome (MERS-CoV) ${ }^{1}$. According to several reports, including those from the Centers for Disease Control and Prevention (CDC), patients with T2DM and the metabolic syndrome might have up to ten-times greater risk of death when they contract COVID-19 (CDC coronavirus reports). Although T2DM and the metabolic syndrome increase the risk of more severe symptoms and mortality in many infectious diseases, there are some additional specific mechanistic aspects in coronavirus infections that require separate consideration, which will have clinical consequences for improved management of patients who are severely affected.

Hyperglycaemia and a diagnosis of T2DM are independent predictors of mortality and morbidity in patients with SARS ${ }^{1}$. This finding could be due to these patients having a state of metabolic inflammation that predisposes them to an enhanced release of cytokines. For COVID-19, a cytokine storm (that is, greatly elevated levels of inflammatory cytokines) has been implicated in the multi-organ failure in patients with severe disease ${ }^{3}$.

Metabolic inflammation will also compromise the immune system, reducing the body's ability to tackle the infection, impairing the healing process and prolonging the recovery. An animal model demonstrated that comorbid T2DM results in immune dysregulation and enhances disease severity following MERS-CoV infection $^{2}$. In this work, diabetic mice expressing the human DPP4 (resulting in MERS-CoV susceptibility) exhibited an altered profile of cytokines, with increased expression of IL-17 $a$ following infection. These data support the hypothesis that the combination of coronavirus infection and T2DM triggers a dysregulated immune response, resulting in a more aggravated and prolonged lung pathology ${ }^{2}$.

\section{A direct endocrine link}

The coronavirus SARS-CoV-2 (which causes COVID-19) enters human cells via the envelope spike glycoprotein, which is also responsible for host-to-host transmission ${ }^{4}$. This glycoprotein, which is found on the surface of the virus, binds to the ectoenzyme angiotensin-converting enzyme 2 (ACE2; located on human cells) to gain entry into the cell. In addition, the cellular serine protease TMPRSS2 is required to prime viral entry via ACE2 ${ }^{4}$.

In the respiratory system, ACE2 has the function of degrading angiotensin II into angiotensin 1-7 and acts as a key regulatory point for the angiotensin system. When ACE1 activity is increased and ACE2 inhibited, intact angiotensin II acts via the angiotensin 1 receptor (AT1R) or AT2R to exert pro-inflammatory responses and stimulate aldosterone secretion; these effects not only increase blood pressure and potentially cause hypokalaemia, but will also increase vascular permeability locally, increasing the risk of respiratory distress syndrome. By contrast, angiotensin 1-7 acts on the Mas receptor pathway, which leads to anti-inflammatory and anti-fibrotic responses that would be favourable to the recovery of patients with COVID- $19^{5}$. It could be postulated that individuals with more severe COVID-19 have an imbalance in the activation of these pathways, with an increase in the activation of AT1R and AT2R, which could be the case in T2DM, hypertension and insulin-resistant states.

\section{A direct metabolic link}

In addition to a link between coronavirus infection and hypertension, there seems to be a direct link to T2DM. In the pancreas, binding of the SARS coronavirus (SARS-CoV, which causes SARS) to its receptor, ACE2, damages islets and reduces insulin release ${ }^{6}$. In one study, patients with SARS who had no history of T2DM and received no steroid treatment were compared with their healthy siblings during a 3-year follow-up period. More than $50 \%$ of the patients in the study became diabetic during hospitalization for the SARS-CoV infection. 
After 3 years of recovery from the viral infection, only $5 \%$ of patients remained diabetic ${ }^{6}$. As the human endocrine pancreas expresses ACE2, the coronavirus might enter islets and cause acute $\beta$-cell dysfunction, leading to acute hyperglycaemia and transient T2DM ${ }^{6}$. More importantly, evidence in diabetic mice demonstrated that ACE2 activity levels were enhanced in the pancreas ${ }^{7}$. This finding suggests that patients with T2DM might be particularly vulnerable to a coronavirus infection. Likewise, T2DM induces expression of angiotensin-converting enzymes in other tissues, including lung, liver and heart ${ }^{7}$, which explains why T2DM can contribute mechanistically to multi-organ failure in SARS-CoV infections.

\section{Immediate clinical consequences}

Based on the data discussed here, it is evident that optimal metabolic control of T2DM and associated metabolic parameters in patients with COVID-19 is mandatory. This is not only relevant because of the obvious danger and increased risk of complications for patients with T2DM and a severe infectious disease, but also because this approach might help the treatment of all patients with COVID-19.

Antidiabetic medications, such as GLP1 agonists, that improve the metabolic function and induce the activity of the protective ACE2 and Mas receptor pathways might have the advantage of ameliorating glucose metabolism and blood pressure, and also preventing coronaviruses from entering cells as a result of competitive binding to ACE2. This effect might help to protect and restore pulmonary function ${ }^{5}$. Likewise, early treatment with angiotensin II receptor blockers (such as losartan or telmisartan) or, more directly, recombinant ACE2, might be useful to enhance the ACE2 and Mas system in preference to the pathways mediated by angiotensin receptors. This approach would enable the combination of an antidiabetic, anti-inflammatory and antiviral effect.

Finally, the synthetic protease inhibitor camustat, which blocks the serine protease TMPRSS2 required for ACE2-mediated coronavirus entry into the cells ${ }^{4}$, also reverses dyslipidaemia and hyperglycaemia ${ }^{8}$. The intriguing link between coronavirus infections and these endocrine and metabolic pathways will have an important effect on the general medical management of severe COVID-19. Glucocorticoids that have been helpful in the treatment of acute respiratory distress syndrome might not be indicated in patients with coronavirus infection. Glucocorticoids not only aggravate metabolic control, but also attenuate angiotensin 1-7 and Mas receptor expression ${ }^{9}$. Hence, they might have a limited role in the management of patients with COVID-19. By contrast, the anti-rheumatic drug hydroxychloroquine, which is now widely used in many centres around the world treating patients with COVID-19, has also attracted interest as a potential therapeutic intervention for patients with $\mathrm{T}_{2} \mathrm{DM}^{10}$. At this point, it is unclear whether hydroxychloroquine in addition to anti-inflammatory and antidiabetic drugs will also directly interfere with the coronavirus-ACE2 pathways.

In conclusion, COVID-19 is not primarily a metabolic disease, but metabolic control of glucose, lipid levels and blood pressure are key in these patients. This approach is important to address the well-established metabolic and cardiovascular complications of this primary comorbidity. Moreover, effective control of these metabolic parameters might represent a specific and mechanistic approach to prevent and ameliorate the acute effects of this virus by reducing the local inflammatory response and blocking its entry into cells.

1. Yang, J. K. et al. Plasma glucose levels and diabetes are independent predictors for mortality and morbidity in patients with SARS. Diabet. Med. 23, 623-628 (2006)

2. Kulcsar, K. A., Coleman, C. M., Beck, S. E. \& Frieman, M. B. Comorbid diabetes results in immune dysregulation and enhanced disease severity following MERS-CoV infection. JCl Insight. 4, 131774 (2019).

3. Mehta, D. et al. Across Speciality Collaboration, UK. COVID-19: consider cytokine storm syndromes and immunosuppression. Lancet https://doi.org/10.1016/S0140-6736(20)30628-0 (2020).

4. Hoffmann, M. et al. SARS-CoV-2 Cell entry depends on ACE2 and TMPRSS 2 and is blocked by a clinically proven protease inhibitor. Cell https://doi.org/10.1016/j.cell.2020.02.05 (2020).

5. Simões e Silva, A. C., Silveira, K. D., Ferreira, A. J. \& Teixeira, M. M ACE2, angiotensin-(1-7) and Mas receptor axis in inflammation and fibrosis. Br. J. Pharmacol. 169, 477-492 (2013).

6. Yang, J. K., Lin, S. S., Ji, X. J. \& Guo, L. M. Binding of SARS coronavirus to its receptor damages islets and causes acute diabetes. Acta Diabetol. 47, 193-199 (2010).

7. Roca-Ho, H., Riera, M., Palau, V., Pascual, J. ¿ Soler, M. J. Characterization of ACE and ACE2 expression within different organs of the NOD mouse. Int. J. Mol. Sci. 18, E563 (2017).

8. Jia, D., Taguchi, M. \& Otsuki, M. Synthetic protease inhibitor camostat prevents and reverses dyslipidemia, insulin secretory defects, and histological abnormalities of the pancreas in genetically obese and diabetic rats. Metabolism. 54, 619-627 (2005).

9. Marshall, A. C. et al. Betamethasone exposure attenuates angiotensin-(1-7)-Mas receptor expression in the dorsal medulla of adult sheep. Peptides 44, 25-31 (2013).

10. Gupta, A. Real-world clinical effectiveness and tolerability of hydroxychloroquine $400 \mathrm{mg}$ in uncontrolled type 2 diabetes subjects who are not willing to initiate insulin therapy (HYQ-Real-World Study). Curr. Diabetes Rev. 15, 510-519 (2019).

\section{Competing interests}

The authors declare no competing interests.

\section{RELATED LINKS}

CDC coronavirus reports: https://www.cdc.gov/mmwr/Novel_Coronavirus Reports.html 